\title{
Spatially resolved In and As distributions in InGaAs/GaP and InGaAs/GaAs quantum dot systems
}

\author{
J. Shen ${ }^{1}$, Y. Song ${ }^{2}$, M. L. Lee ${ }^{2}$, and J. J. Cha ${ }^{1,3}$ \\ 1. Department of Mechanical Engineering and Materials Science, Yale University, New Haven, CT, \\ USA \\ 2. Department of Electrical Engineering, Yale University, New Haven, CT, USA \\ 3. Energy Science Institute, Yale University West Campus, West Haven, CT, USA
}

InGaAs/GaP QD self-assembled quantum dot (QD) systems are of tremendous interest, for GaP can be directly grown on $\mathrm{Si}$, enabling true monolithic integration of optoelectronics with $\mathrm{Si}$ technology [1]. Promising optical properties such as light emitting diodes (LEDs) at room temperature [2] and lasing at $80 \mathrm{~K}$ [3] have been demonstrated in these systems. It is critical that we develop a detailed microscopic understanding of the QD structures and elemental distributions of InGaAs/GaP QD systems because the band structure and band alignment, and thus the optical properties, depend on the detailed atomic structure of the QDs and spatial distributions of each element (In, Ga, As) [4-6], particularly In [7].

So far, two-beam TEM imaging has been the popular choice to image these QDs (Figure 1). In addition, the In distribution in these QDs has been inferred from lattice constant changes or diffraction contrast in TEM [8] or STM [9]. These indirect measurements can be potentially inaccurate due to factors such as strain relaxations in thin TEM samples and other elements (As, Ga, or P) affecting the lattice constant. Low-loss electron energy loss spectroscopy (EELS) was used to report the elemental distribution [10], but careful post subtraction of the zero loss peak is necessary. Therefore, direct measurements of In, Ga, and $\mathrm{As}$ in InGaAs/GaAs and InGaAs/GaP QD systems are highly desired.

We directly measure two-dimensional (2D) inhomogeneous distributions of In and As in InGaAs QDs grown in the GaAs and GaP matrix, using energy-dispersive X-ray spectral imaging in a scanning transmission electron microscope (EDX-STEM). The 2D chemical maps were acquired within several minutes, made possible by the combination of the high brightness electron source and high-solid-angle area, quadrant EDX detector, dramatically increasing the signal to noise ratio of the EDX maps.

Our main finding is that, in InGaAs/GaP QD systems, the In elemental distribution does not closely follow the QD structures while the As profile does (Figure 2). In contrast, for InGaAs/GaAs QD systems, In is concentrated within the QDs and along the interfacial wetting layer (Figure 2). Comparing the InGaAs/GaAs (Figure 2j-m) and InGaAs/GaP (Figure 2c-g) QD systems, In is more broadly distributed in the GaP matrix. Our findings show that the In elemental profile is much more complicated than previously assumed and that replacing the GaAs matrix with $\mathrm{GaP}$ for monolithic integration with $\mathrm{Si}$ results in different elemental profiles of InGaAs QDs. These findings challenge our current understanding of the band structure model of InGaAs/GaP QD systems [4, 6]. The detailed analysis of elemental distributions of InGaAs QDs on GaAs and GaP gives us a better understanding of the atomic structure of the InGaAs QDs, which can lead to improved QD structures and better optical properties by optimizing the growth parameters. 
Reference:

[1] Volz, K. et al. Journal of Crystal Growth 2011, 315, 37-47.Roesener, T. et al. Journal of Crystal Growth 2013, 368, 21-28.

[2] Song, Y.; Larry Lee, M. Applied Physics Letters 2013, 103, 141906.

[3] Heidemann, M. et al. Applied Physics Letters 2014, 104, 011113.

[4] Nguyen Thanh, T., et al. Applied Physics Letters 2011, 99, 143123.

[5] Fukami, F. et al. physica status solidi (c) 2011, 8, 322-324. Shumway, J. et al. Physical Review B 2001, 64, 125302.

[6] Fuchi, S. et al. Physica E: Low-dimensional Systems and Nanostructures 2004, 21, 36-44.

[7] Liu, N. et al. Physical Review Letters 2000, 84, 334-337.Liao, X. Z. et al. Physical Review Letters 1999, 82, 5148-5151.Rosenauer, A. et al. Applied Physics Letters 1997, 71, 3868-3870.

[8] Ruvimov, S.; Scheerschmidt, K. physica status solidi (a) 1995, 150, 471-478.Guha, S. et al. Applied Physics Letters 1990, 57, 2110-2112.

[9] Flebbe, O. et al. Journal of Vacuum Science \& Technology B 1999, 17, 1639-1648.Prohl, C., et al. Applied Physics Letters 2013, 102, 123102. Vegard, L. Z. Physik 1921, 5, 17-26.

[10] Molina, S. I., et al. Applied Physics Letters 2007, 91, 263105.
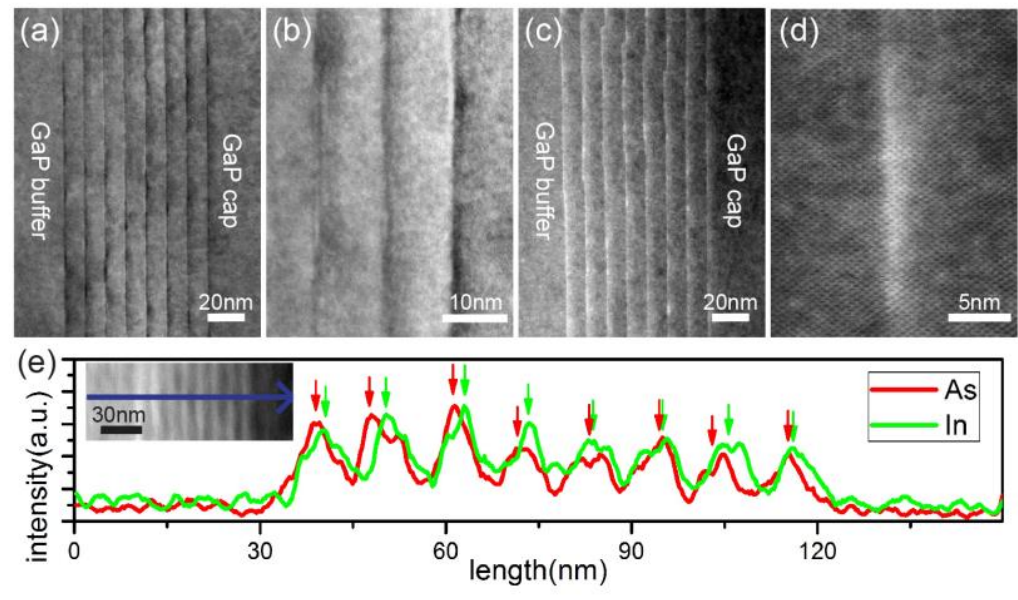
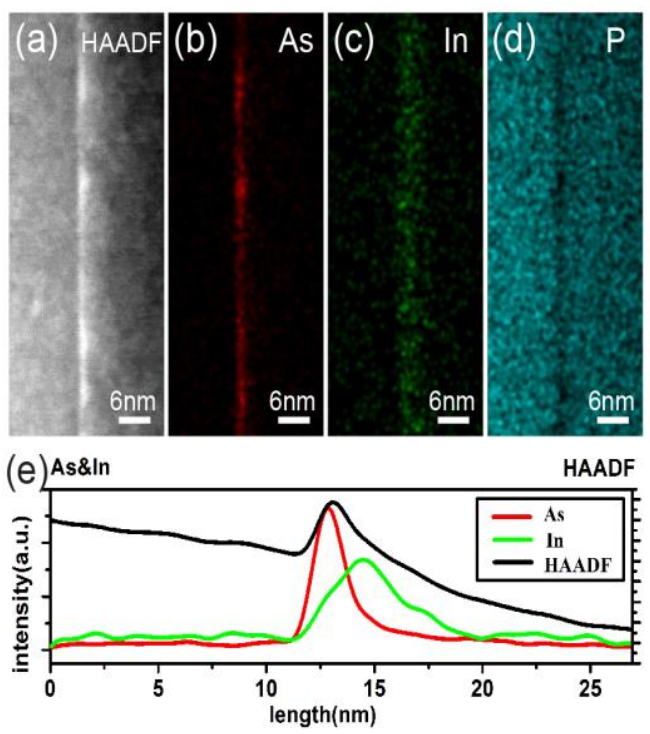
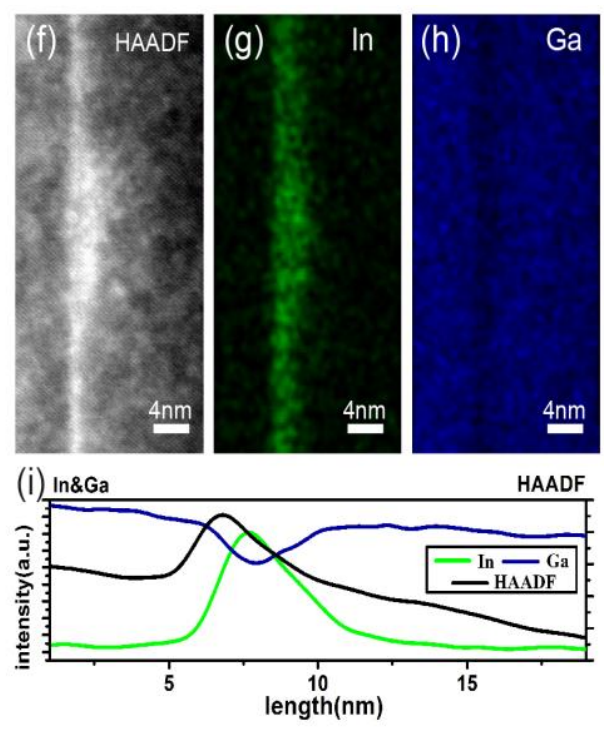

Figure 1. Two-beam (002) and HAADF-STEM imaging of 8 stacks of InGaAs/GaP QDs grown on $\mathrm{GaP} / \mathrm{Si}$. (a) and (b) show two-beam (002) TEM images where the dark regions point to QD locations due to local strain. (c) and (d) show HAADF-STEM images of the same sample. (e) shows elemental line profiles of As and In across the 8 stacks of QD layers. 